\title{
MAXIMIZATION OF TIME-TO-FIRST-FAILURE FOR MULTICASTING IN WIRELESS NETWORKS : OPTIMAL SOLUTION
}

\author{
Arindam K. Das, Mohamed El-Sharkawi \\ Department of Electrical Engineering \\ University of Washington, Box 352500, Seattle, WA 98195. \\ email: \{arindam,elsharkawi\}@ee.washington.edu \\ Robert J. Marks \\ Rogers Engineering and Computer Science Building \\ Baylor University, 1311 S. 5th Street, Waco, TX 76798-7356. \\ email: Robert_Marks@Baylor.edu \\ Payman Arabshahi, Andrew Gray \\ Jet Propulsion Laboratory \\ 4800 Oak Grove Drive, MS 238-343, Pasadena, CA 91109. \\ email: \{payman,gray\}@jpl.nasa.gov
}

\begin{abstract}
We consider the problem of maximizing the time-to-firstfailure (TTFF), defined as the time till the first node in the network runs out of battery energy, in energy constrained broadcast wireless networks. We show that the TTFF criterion, by itself, fails to provide the "ideally optimum" multicast tree and propose a composite weighted objective function which maximizes the TTFF and minimizes the sum of transmitter powers. We then develop a mixed integer linear programming (MILP) model for solving the joint optimization problem optimally. We also consider the case of prioritized nodes and show how the model can be modified to deal with such priorities.
\end{abstract}

\section{INTRODUCTION}

We consider the problem of maximizing the time-to-firstfailure in energy constrained broadcast wireless networks where each node is powered by batteries. In applications where replacement/maintenance of such batteries is difficult or infeasible, it is of utmost importance to design routing protocols which maximize the lifetime of the network. A metric commonly used to define the lifetime of a network is the duration of time before any node in the network runs out of its battery energy. We define this time to be the time-to-firstfailure (TTFF), also known as system lifetime or network lifetime in the literature. To the best of our knowledge, this problem was first addressed by Chang and Tassiulas for an unicast

\footnotetext{
This work was supported by the Advanced Information Systems Technology (AIST) program at the NASA Offi ce of Earth Sciences.
}

application [1]. Subsequent research in this area for unicast as well as multicast applications have been reported in [2], [3], [4] and [5]. In [7], it is shown that maximization of the TTFF for a broadcast application can be solved optimally by a greedy algorithm in polynomial time.

In this paper, we first illustrate that simply optimizing the TTFF criterion may not provide the "best possible" solution. This motivates the use of a composite objective function involving the sum of the transmitter powers. We then present a mixed integer linear programming (MILP) model for solving the joint optimization problem optimally. The MILP model is based on the well-studied single-origin multiple-destination uncapacitated flow problem, tailored to reflect the inherently broadcast nature of the wireless medium. Finally, we consider the case of prioritized nodes and show how the model can be modified to deal with such priorities.

\section{NETWORK MODEL}

We assume a fixed $N$-node network with a specified source node which has to broadcast a message to all other nodes in the network. Any node can be used as a relay node to reach other nodes in the network. All nodes are assumed to have omni-directional antennas, so that if node $i$ transmits to node $j$, all nodes closer to $i$ than $j$ will also receive the transmission (provided line-of-sight exists).

We assume that, for a transmission from node $i$ to $j$, the received signal power at $j$ varies as $d_{i j}^{-\alpha}$, where:

$$
d_{i j}=\left[\left(x_{i}-x_{j}\right)^{2}+\left(y_{i}-y_{j}\right)^{2}\right]^{1 / 2}
$$


is the Euclidean distance between nodes $i$ and $j,\left(x_{i}, y_{i}\right)$ are the coordinates of node $i$ and $\alpha$ (typically in the range $2 \leq \alpha \leq 4$ ) is the channel loss exponent. Consequently, the transmitter power at $i$ necessary to support the link $i \rightarrow j$, $\mathbf{P}_{i j}$, is proportional (accounting for fading and antenna gain factors) to $d_{i j}^{\alpha}$. Without any loss of generality, we set the proportionality constant to be equal to 1 and therefore:

$$
\mathbf{P}_{i j}=d_{i j}^{\alpha}
$$

The power matrix of a network, $\mathbf{P}$, is defined to be an $N \times N$ symmetric matrix whose $(i, j)$ th element, $\mathbf{P}_{i j}$, represents the power required to support the link $i \rightarrow j$.

Finally, we assume that power expenditures due to signal reception and processing are negligible compared to signal transmission and hence the lifetime is determined solely by the choice of transmitter powers and residual energy levels of the nodes.

\section{Problem Statement}

Let $E(t)$ be a vector of node residual energies at time $t$, the $i t h$ element of $E(t)$ representing the residual energy of node $i$ at time $t$, and $Y$ be a vector of node transmission powers. The element $Y_{i}$ represents the transmitter power level of node $i$. We assume that each node has a constraint on maximum transmitter power, denoted by $Y_{i}^{\max }$. That is:

$$
Y_{i} \leq Y_{i}^{\max }: \forall i \in \mathcal{N}
$$

where $\mathcal{N}$ is the set of all nodes in the network.

Also, let $s$ be the source, $\mathcal{D}$ the set of destination nodes and $\mathcal{E}$ the set of all directed edges ${ }^{1}$ and $\mathcal{D}$ the set of destination nodes, $\mathcal{D} \subseteq\{\mathcal{N} \backslash s\}$. Let the cardinality of these sets be $N$, $E$ and $D$ respectively; i.e., $N=|\mathcal{N}|, E=|\mathcal{E}|$ and $D=|\mathcal{D}|$. Using the transmitter power constraint, the set of all edges, $\mathcal{E}$, is given by:

$$
\mathcal{E}=\left\{(i \rightarrow j):(i, j) \in \mathcal{N}, i \neq j, \mathbf{P}_{i j} \leq Y_{i}^{\max }, j \neq s\right\}
$$

The third condition in the right hand side of (3) specifies the set of nodes reachable by a direct transmission from any transmitting node depending on its power constraint. The last condition reflects that no transmitting node needs to reach the source node.

Defining $L_{i}(t) \triangleq E_{i}(t) / Y_{i}$ to be the lifetime of node $i$, the problem of maximizing the TTFF can be written as:

$$
\operatorname{maximize}\left\{\min _{i \in \mathcal{N}} L_{i}(t)\right\}
$$

\footnotetext{
${ }^{1}$ In this paper, we assume that all edges are directed. The notation $(i \rightarrow$ $j$ ) will be used to denote a directed edge from node $i$ to $j$. The notation $(i, j)$ will be used to refer to the node pair.
}

The objective function in (4) is to be optimized subject to the following constraints:

1) All nodes, other than the source, must be reached, either actually or implicitly ${ }^{2}$.

2) The source node must reach at least one other node.

3) The solution must be a connected tree; i.e., there must be directed paths from the source to all destination nodes, possibly involving other intermediate nodes.

The vector $\vec{L}(t) \triangleq\left\{L_{i}(t): \forall i \in \mathcal{N}\right\}$ is the node lifetime vector at time $t$. Note that the value of the expression within curly braces in (4) is dependent on the time index $t$ and hence, strictly speaking, should be termed residual-timeto - first - failure. However, we will refer to it simply as the time-to-first-failure, implicitly recognizing its dependence on the time origin $t$. Accordingly, henceforth in this paper, we will simply use the notations $E_{i}$ and $L_{i}$ instead of $E_{i}(t)$ and $L_{i}(t)$.

Assuming that all nodes in the network have omnidirectional antennas, a transmission from node $i$ to node $j$ would also be received by all nodes geometrically closer to $i$ than $j$. Let $S$ be the set of nodes that are geometrically closer to $i$ than $j\left(\Rightarrow \mathbf{P}_{i j}>\mathbf{P}_{i k}: \forall k \in S\right)$. Nodes that belong in $S$ are said to receive the transmission from $i$ implicitly (in the sense that no additional cost is incurred to reach them) and the set of transmissions $\{i \rightarrow k: \forall k \in S\}$ are referred to as implicit transmissions. The transmission $i \rightarrow j$ is referred to as an actual transmission.

Let $\left\{X_{i j}:(i \rightarrow j) \in \mathcal{E}\right\}$ be a set of binary variables such that $X_{i j}=1$ if the transmission $i \rightarrow j$ is used in the optimum tree and 0 otherwise. Following our discussion in the previous paragraph, we can write:

$$
Y_{i}=\max _{j}\left\{X_{i j} \mathbf{P}_{i j}: j \neq i\right\}
$$

where $X_{i j}=1$ if node $j$ is reached from node $i$ (actually or implicitly) and 0 otherwise. Note that equation (5) is a direct consequence of our assumption of omni-directional antennas and implies that the cost of spanning in multiple nodes from node $i$ is simply the cost incurred in reaching the farthest node.

We now express the objective function in (4) as a minimax

\footnotetext{
${ }^{2}$ Note that the possibility of reaching a node implicitly is a consequence of the inherently broadcast nature of the wireless network and our assumption of omni-directional antennas.
} 
optimization problem as follows:

$$
\begin{aligned}
& \text { maximize }\left(\min _{i} L_{i}\right) \\
& =\text { maximize }\left(\min _{i} E_{i} / Y_{i}\right) \\
& =\text { minimize }\left(\max _{i} Y_{i} / E_{i}\right) \\
& =\operatorname{minimize}\left(\max _{i}\left[\max _{j}\left(\mathbf{P}_{i j} X_{i j}\right) / E_{i}\right]\right) \\
& =\text { minimize }\left(\max _{i, j}\left[\mathbf{P}_{i j} X_{i j} / E_{i}\right]\right) \\
& =\text { minimize } \sigma
\end{aligned}
$$

where

$$
\sigma=\max _{i, j}\left(\mathbf{P}_{i j} X_{i j} / E_{i}\right)=1 / \tau
$$

and $\tau$ is the TTFF.

We conclude this section with definitions of critical node and critical transmission. For a given connection tree, $T$, we define its critical node to be the node whose residual lifetime is equal to the TTFF of the tree. That is:

$$
\text { Critical node }=\operatorname{argmin}_{i}\left(E_{i} / Y_{i}\right)
$$

Note that for any non-transmitting node, $Y_{i}=0$, and hence the residual lifetime of that node is $\infty$.

A transmission $(i \rightarrow j)$ is defined to be the critical transmission in a tree if:

$$
E_{i} / \mathbf{P}_{i j}=\mathrm{TTFF} \triangleq \min _{i}\left(E_{i} / Y_{i}\right)
$$

In the next section, we illustrate with an example the inadequacy of the TTFF criterion when optimized singly. This will motivate the need for a joint objective function involving the sum of transmitter powers. In Section V, we develop a mixed integer linear programming model for the joint optimization problem.

\section{INADEQUACY OF THE TTFF CRITERION}

Consider the 6-node network and the broadcast tree in Figure 1a. Assuming $\alpha=2$, the power matrix of the network is:

$$
\mathbf{P}=\left[\begin{array}{rrrrrr}
0 & 14.86 & 9.31 & 6.33 & 7.01 & 1.76 \\
14.86 & 0 & 23.18 & 4.39 & 4.58 & 6.46 \\
9.31 & 23.18 & 0 & 7.41 & 24.32 & 11.65 \\
6.33 & 4.39 & 7.41 & 0 & 7.11 & 2.73 \\
7.01 & 4.58 & 24.32 & 7.11 & 0 & 2.43 \\
1.76 & 6.46 & 11.65 & 2.73 & 2.43 & 0
\end{array}\right]
$$

Assume that the residual energy of all nodes is 10 . The residual lifetime vector of the nodes for the tree in Figure 1a is: $\vec{L}_{1}=[\infty, 1.55, \infty, 1.35, \infty, 5.69]$. The lifetimes of nodes 1 , 3 and 5 are $\infty$ since they are non-transmitting nodes in the tree. Node 4 is the critical node in the tree and $4 \rightarrow 3$ is the critical transmission.
Now consider the broadcast tree in Figure $1 b$. The residual lifetime vector in this case is: $\vec{L}_{2}=$ $[\infty, 2.28, \infty, 1.35, \infty, \infty]$. The TTFF of this tree is identical to that of Figure 1a. However, note that the lifetime of node 2 is higher (2.28, as compared to 1.55 ) than its lifetime in Figure 1a. Also, the lifetime of node 6 is now $\infty$, compared to 5.69 in Figure 1a, since it is a non-transmitting node. Clearly, for the same TTFF, this broadcast tree is better than that shown in Figure 1a.

In general, given two trees $T_{m}$ and $T_{n}$ with the same TTFF, $T_{m}$ is considered better ("leaner") than $T_{n}$ if:

- there is at least one node in $T_{m}$ whose residual lifetime is greater in $T_{m}$ than in $T_{n}$, and,

- the residual lifetimes of all other nodes in $T_{m}$ are at least as high as in $T_{n}$.

One way of obtaining a "lean" optimum solution is to consider a joint optimization function of the form:

$$
\text { minimize } w_{1} \sigma+w_{2} \sum_{i=1}^{N} Y_{i}
$$

where $\sum_{i=1}^{N} Y_{i}$ is the sum of transmitter powers, $\sigma$ is the inverse of the TTFF (10) and $\left\{w_{1}, w_{2}\right\}$ are suitably chosen non-negative penalty factors.

It can be easily verified that the tree in Figure $1 \mathrm{~b}$ is characterized by a smaller total transmitter power, 11.80 units $\left(P_{24}+P_{43}\right)$, compared to the tree in Figure 1a which uses a total transmitter power of 15.63 units $\left(P_{26}+P_{61}+P_{43}\right)$.

The first parameter in (14), $\sigma$, may be viewed as the global cost while the second parameter, $\sum_{i=1}^{N} Y_{i}$, may be viewed as the sum of local costs. Varying $w_{1}$ and $w_{2}$ in (14) therefore represents a tradeoff between the global and local costs. Although not a focus of this paper, we would like to point out that trading off $w_{1}$ versus $w_{2}$ also affects the number of hops in the optimal solution, a critical criterion by itself for certain military applications, since using a large number of hops increases the probability of detection/interception by enemy radar. In general, the optimal tree for $w_{2}=0$ uses a far more number of hops - consequently, incurring higher average path delay - than the optimal tree for $w_{1}=0$.

Note that for the special case of $w_{2}=0$, an optimal polynomial time algorithm exists, as discussed in [7]. It is interesting to note that, if the residual energies of the nodes are identical (say, $E$ ), the objective function reduces to a "minimization of the maximum transmitter power" problem which can also be solved optimally in polynomial time, being a special case of an arbitrary residual energy vector.

For $w_{2}>0$, however, it is unlikely that any optimal polynomial time algorithm exists, since the problem of minimiz- 
ing the sum of transmitter powers $\left(w_{1}=0\right.$ in (14)) has been shown to be NP-complete [8].

Note that for the special case of equal residual energies and $w_{1}, w_{2} \neq 0$, the problem reduces to a joint minimization of "maximum transmitter power and sum of transmitter powers".

In this paper, we are concerned primarily with the case when $w_{1}>>w_{2} \neq 0$. For a proper choice of these parameter values, it is possible to obtain the "best possible" tree which maximizes the TTFF while ensuring that the solution is the most power efficient among the set of all trees with optimal TTFF. The concept of using a secondary optimization criterion, such as sum of transmitter powers, is not new and was mentioned by Ramanathan and Rosales-Hain in the context of topology control of wireless ad-hoc networks [6]. It should be noted that using the most power efficient optimal TTFF tree also helps to control the total interference power in the system.

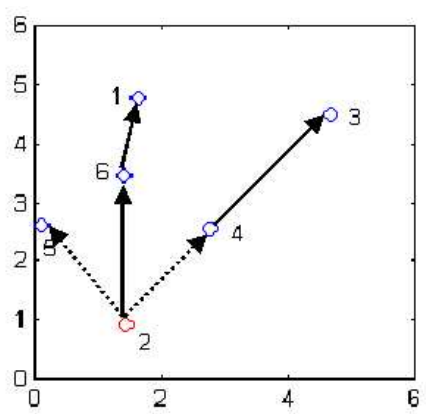

Fig. 1a. The residual energy of each of the nodes is 10 . The power matrix of the network is given in (13). TTFF of the broadcast tree $\{2 \rightarrow 6,6 \rightarrow$ $1,4 \rightarrow 3\}$ is 1.35 , node 4 being the critical node. The node lifetime vector corresponding to the tree is: $[\infty, 1.55, \infty, 1.35, \infty, 5.69]$. The lifetimes of nodes 1,3 and 5 are $\infty$ since they are non-transmitting nodes in the tree. Note that the transmissions $2 \rightarrow 4$ and $2 \rightarrow 5$ are implicit, since nodes 4 and 5 are nearer to 2 than 6 .

\section{MILP MODEL}

In this section, we develop a mixed integer linear programming model of the joint optimization problem involving the TTFF criterion and the sum of the transmitter powers (14).

Let $\left\{F_{i j}: \forall(i \rightarrow j) \in \mathcal{E}\right\}$ be a set of flow variables $\left(F_{i j}\right.$ represents the flow from node $i$ to node $j$ ), with $\mathcal{E}$ defined as in (3). The general multicast problem can be interpreted as a single-origin multiple-destination uncapacitated flow problem, with the source (the supply node) having $D$ units of supply and the destination nodes (demand nodes) having one unit of demand each. For other nodes, the net in-flow must equal the net out-flow, since they serve only as relay nodes. At a conceptual level, the flow model can be viewed

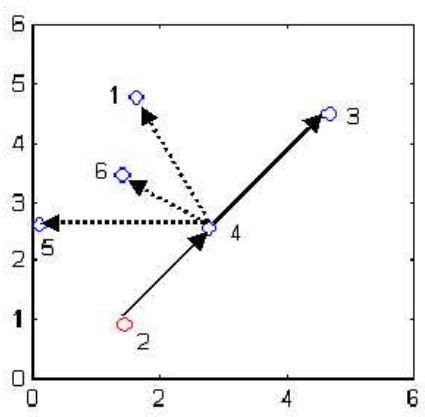

Fig. 1b. An alternate broadcast tree with the same TTFF, 1.35, as in Figure 1a. In this tree, the transmissions $4 \rightarrow 1,4 \rightarrow 5$ and $4 \rightarrow 6$ are implicit. The node lifetime vector is: $[\infty, 2.28, \infty, 1.35, \infty, \infty]$. Note that the lifetime of node 2 is higher (2.28, as compared to 1.55) than its lifetime in Figure 1a. Also, the lifetime of node 6 is now $\infty$, compared to 5.69 in Figure 1a, since it is a non-transmitting node. Clearly, for the same TTFF, this broadcast tree is better than that shown in Figure 1a.

as a token allocation scheme where the source node generates as many tokens as there are destination nodes and distributes them along the "most efficient" tree such that each destination node gets to keep one token each. For example, for the broadcast tree in Figure 1b, the flow variables are $F_{24}=5, F_{41}=F_{43}=F_{45}=F_{46}=1$, the rest of the variables being 0 .

The single-origin multiple-destination flow problem discussed above can be solved using the usual conservation of flow constraints as shown below (see [9] for example):

$$
\begin{gathered}
\sum_{j=1}^{N} F_{i j}=D ; i=s,(i \rightarrow j) \in \mathcal{E} \\
\sum_{j=1}^{N} F_{j i}-\sum_{j=1}^{N} F_{i j}=1 ; \forall i \in \mathcal{D},(i \rightarrow j) \in \mathcal{E} \\
\sum_{j=1}^{N} F_{j i}-\sum_{j=1}^{N} F_{i j}=0 ; \forall i \notin\{\mathcal{D} \cup s\},(i \rightarrow j) \in \mathcal{E}
\end{gathered}
$$

Having set up the flow equations, we now have to write down constraints linking the flow variables to the power variables, $\left\{Y_{i}\right\}$. We do this in two stages. In the first stage, we couple the flow variables and the indicator variables $\left\{X_{i j}\right\}$ and in the next stage, we link the $\left\{X_{i j}\right\}$ variables to the power variables. Recall from Section III that $X_{i j}=1$ if the edge $i \rightarrow j$ appears in the optimum solution (either as an actual transmission or as an implicit transmission) and 0 otherwise.

The set of constraints which couple the flow variables and the $X_{i j}$ variables are:

$$
D \cdot X_{i j}-F_{i j} \geq 0 ; \forall(i \rightarrow j) \in \mathcal{E}
$$

where $D$ is the number of destination nodes. Note that (18) 
ensures that " $X_{i j}=1$ if $F_{i j}>0$ ". The coefficient of $X_{i j}$ in (18) is due to the fact that the maximum flow out of any node on a single link is equal to the number of destination nodes. Equation (18), however, leaves open the possibility of $X_{i j}$ being equal to 1 for $F_{i j}=0$. We show later that, for $w_{2}>0$ (equation 14), doing so would unnecessarily increase the cost of the optimum solution and therefore this possibility can be discounted. For the broadcast tree in Figure $1 b$, the status of the $X_{i j}$ variables are $X_{24}=X_{41}=X_{43}=X_{45}=X_{46}=1$, the rest being 0 . It should also be noted that the smallest integer value of $X_{i j}$ which satisfies (18) for any nonzero flow out of node $i$ (i.e., $\sum_{j} X_{i j} \geq 1$ ) is 1 . Consequently, we can simply define the $X_{i j}$ 's to be integers, instead of explicitly declaring them to be binary variables.

Next, we write down constraints linking the $X_{i j}$ variables and the power variables. As discussed in Section III (see eqn. 5 ), for an omni-directional antenna system, the cost of spanning in multiple nodes from node $i$ is simply the cost incurred in reaching the farthest node. This condition is expressed as:

$$
Y_{i}-\mathbf{P}_{i j} X_{i j} \geq 0 ; \forall i \in \mathcal{N}, \forall(i \rightarrow j) \in \mathcal{E}
$$

In order to relate the inverse TTFF parameter, $\sigma$, to the power variables, we note that $\sigma=\max _{i} Y_{i} / E_{i}$ (compare equations 6 and 9). As in (19), this condition can be written as:

$$
\sigma-Y_{i} / E_{i} \geq 0 ; \forall i \in \mathcal{N}
$$

It is now clear that for $w_{2}>0$, if there is no flow out of node $i$ (i.e., $\sum_{j} F_{i j}=0$ ), setting $X_{i j}=1$ would result in a positive value for $Y_{i}$ and thereby unnecessarily increase the cost of the optimal solution.

So far, we have implicitly assumed that the residual lifetimes of all transmitting nodes are greater than the multicast duration $^{3}$. In other words, if $L$ is the total number of bits to be transmitted during the session and $R$ is the data rate in bps (assumed uniform throughout the network), we have assumed that:

$$
E_{i} / Y_{i} \geq L / R \Longleftrightarrow Y_{i} / E_{i} \leq R / L
$$

Constraints of the form (21) can be explicitly added to the model to ensure that all nodes choose transmitter power levels such that their residual lifetimes are greater than or equal to the multicast session duration, $L / R$.

The final set of constraints express the integrality of the $X_{i j}$ variables and non-negativity of the $F_{i j}$ and $Y_{i}$ variables. We note that the number of integer variables is equal to $E$

\footnotetext{
${ }^{3}$ We assume static multicasting; i.e., the same tree is used for the entire multicast duration.
}

while the number of continuous variables is equal to $E+N$. The number of constraints is equal to $2 E+3 N$.

$$
\begin{aligned}
X_{i j} & \geq 0, \text { integer; } \forall i \in \mathcal{N} \\
F_{i j} & \geq 0 ; \quad \forall(i \rightarrow j) \in \mathcal{E} \\
Y_{i} & \geq 0 ; \quad \forall i \in \mathcal{N}
\end{aligned}
$$

\section{A. Dealing with prioritized nodes}

The MILP model we discussed above assumes that all nodes enjoy equal priority in the network. We now consider the case where nodes may have unequal priorities, e.g., depending on their location in the grid ${ }^{4}$ or on their residual energies. Let $b_{i}$ be the priority associated with node $i$, $0<b_{i} \leq 1$. The effective lifetime ${ }^{5}$ of node $i, L_{i}^{e f f}$, is now defined as:

$$
L_{i}^{e f f}=E_{i} / b_{i} Y_{i}
$$

Consequently, we redefine the inverse TTFF parameter as follows (instead of eqn. 10):

$$
\sigma=\max _{i}\left(b_{i} Y_{i} / E_{i}\right)=1 / \tau
$$

The above equation can be expressed as the following set of linear constraints:

$$
\sigma-b_{i} Y_{i} / E_{i} \geq 0 ; \forall i \in \mathcal{N}
$$

Solving the optimization problem with (27) instead of (20) yields a node prioritized optimum solution. We illustrate the concept of node weighting with an example.

Consider the 3-node network in Figure 2. Assume $\mathbf{P}_{A B}=$ $2, \mathbf{P}_{B C}=1.5, \mathbf{P}_{A C}=5, E_{A}=10$ and $E_{B}=5$. Let $b_{A}=b_{B}=1$. Under these conditions, the optimal TTFF broadcast tree, considering node $A$ to be the source, is $\{A \rightarrow B, B \rightarrow C\}$, with a TTFF of $10 / 3$ (node $B$ is the critical node). If, however, $b_{A}=0.5$ and $b_{B}=1$ (i.e., it is more important to preserve node $B$ than $A$ ), it can be easily verified that the optimization process yields the broadcast tree $\{A \rightarrow C\}$, with node $B$ reached implicitly. Note that the effective lifetime of node $A$, as computed by the optimization process, is $E_{A} / b_{A} \mathbf{P}_{A C}=10 /(0.5 \times 5)=4$ but its actual lifetime is $E_{A} / \mathbf{P}_{A C}=10 / 5=2$. This example illustrates how node $B$ can be preserved, at the expense of node $A$, by assigning suitable node weights.

Figure 3 summarizes the generalized MILP model, with arbitrary node weights.

\footnotetext{
${ }^{4}$ For example, barycentric nodes may be assigned higher priorities to prevent their premature burn-out.

${ }^{5}$ Note that the actual lifetime of node $i$ is still given by $E_{i} / Y_{i}$. The notion of effective lifetime is used only to guide the optimization process to choose a tree avoiding the nodes accorded the highest priorities, if one exists, as illustrated subsequently.
} 


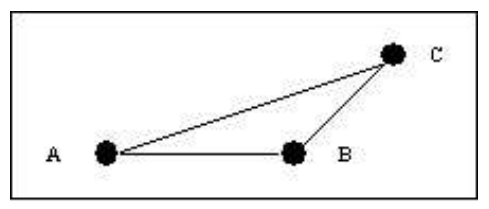

Fig. 2. An example 3-node network.

$$
\text { minimize } w_{1} \sigma+w_{2} \sum_{i=1}^{N} Y_{i}
$$

subject to:

$$
\begin{aligned}
& \sigma-b_{i} Y_{i} / E_{i} \geq 0 ; \forall i \in \mathcal{N} \\
& R / L-Y_{i} / E_{i} \geq 0 ; \forall i \in \mathcal{N} \\
& Y_{i}^{\text {max }}-Y_{i} \geq 0 ; \forall i \in \mathcal{N} \\
& Y_{i}-\mathbf{P}_{i j} X_{i j} \geq 0 ; \forall i \in \mathcal{N}, \forall(i \rightarrow j) \in \mathcal{E} \\
& D \cdot X_{i j}-F_{i j} \geq 0 ; \forall(i \rightarrow j) \in \mathcal{E} \\
& \sum_{j=1}^{N} F_{i j}-D=0 ; i=\operatorname{source},(i \rightarrow j) \in \mathcal{E} \\
& \sum_{j=1}^{N} F_{j i}-\sum_{j=1}^{N} F_{i j}-1=0 ; \forall i \in \mathcal{D},(i \rightarrow j) \in \mathcal{E} \\
& \sum_{j=1}^{N} F_{j i}-\sum_{j=1}^{N} F_{i j}=0 ; \forall i \notin \mathcal{D}, i \neq \text { source },(i \rightarrow j) \in \mathcal{E} \\
& X_{i j} \geq 0, \text { integer } ; \forall(i \rightarrow j) \in \mathcal{E} \\
& F_{i j} \geq 0 ; \forall(i \rightarrow j) \in \mathcal{E} \\
& Y_{i} \geq 0 ; \forall i \in \mathcal{N}
\end{aligned}
$$

Fig. 3. Generalized MILP model for the joint minimization of inverse TTFF and sum of transmitter powers with arbitrary node weights.

\section{Conclusion}

In this paper, we have considered the problem of maximizing the time-to-first-failure in broadcast wireless networks. We showed that simply maximizing the TTFF (or, minimizing the inverse TTFF) criterion may not yield the best possible solution. This motivated us to consider a joint optimization problem involving the TTFF criterion and a secondary criterion such as the sum of transmitter powers. Finally, we presented a mixed integer linear programming model for solving the joint optimization problem and showed how the model can be modified to deal with prioritized nodes.

Currently, we are conducting extensive network simula- tions to quantify the effect of trading off total transmit power versus maximum transmit power on performance parameters such as throughput and end-to-end delay. Preliminary results confirm our intuition that multicast trees designed to minimize the maximum transmit power generally suffer from reduced throughput and higher latencies as the network load increases, compared to multicast trees which minimize the total transmit power. These results would be presented in an upcoming paper.

\section{REFERENCES}

1) Jae-Hwan Chang and Leandros Tassiulas, "Energy Conserving Routing in Wireless Ad-Hoc Networks", Proc. of INFOCOM, 2000.

2) R. J. Marks II, A. K. Das, M. A. El-Sharkawi, P. Arabshahi and A. Gray, "Maximizing Lifetime in an Energy Constrained Wireless Sensor Array Using Team Optimization of Cooperating Systems", Proc. of the World Congress on Computational Intelligence, Honolulu, Hawaii, 2002.

3) Archan Misra and Suman Banerjee, "MRPC: Maximizing Network Lifetime for Reliable Routing in Wireless Environments", Proc. of WCNC, 2002.

4) Intae Kang and Radha Poovendran, "On the Lifetime Extension of Energy-Efficient Multihop Broadcast Networks", Proc. of the World Congress on Computational Intelligence, Honolulu, Hawaii, 2002.

5) C.K. Toh, "Maximum Battery Life Routing to support ubiquitous Mobile Computing in Wireless Ad Hoc Networks", IEEE Communications Magazine, June 2001.

6) Ram Ramanathan and Regina Rosales-Hain, "Topology Control of Multihop Wireless Networks using Transmit Power Adjustment", Proc. INFOCOM, 2000.

7) A. K. Das, R.J. Marks II, M.A. El-Sharkawi, P. Arabshahi and A. Gray, "MDLT: A Polynomial Time Optimal Algorithm for maximization of Time-to-FirstFailure in Energy-Constrained Broadcast Wireless Networks," Proc. GLOBECOM, San Francisco, December 1-5, 2003.

8) M. Cagalj, J.P. Hubaux, C. Enz, "Minimum-energy broadcast in all-wireless networks: NP-completeness and distribution issues," Proc. MOBICOM, Sep. 2002, Atlanta, Georgia, USA.

9) Richard Kipp Martin, "Large Scale Linear and Integer Optimization: A Unified Approach”, Kluwer Academic Publishers, Massachusetts, 1999. 